\title{
DISEÑO Y APLICACIÓN EDUCATIVA DE UN PROGRAMA DE SIMULACIÓN PARA EL APRENDIZAJE DE TÉCNICAS EXPERIMENTALES CON SISTEMAS DE ADQUISICIÓN DE DATOS
}

\author{
Alfonso Pontes, Jorge Gavilán, Marta Obrero y Andrés Flores \\ Escuela Politécnica Superior de la Universidad de Córdoba
}

[Recibido en julio de 2005, aceptado en Noviembre de 2005]

\begin{abstract}
RESUMEN(Inglés)
En este trabajo se describe una experiencia educativa sobre el uso de las tecnologías de la información y la comunicación en el aprendizaje de técnicas experimentales, que es un tema importante para el desarrollo de prácticas de laboratorio de Física, en las carreras universitarias de ciencias e ingeniería. La experiencia se ha centrado en diseñar y utilizar un programa de simulación que pretende mejorar el aprendizaje de procedimientos científicos, relacionados con la recogida $y$ análisis de datos experimentales, utilizando un sistema informático de adquisición de datos que se conecta en cada experiencia a varios sensores físicos.
\end{abstract}

Palabras clave: Aprendizaje de técnicas y destrezas científicas; laboratorio asistido por ordenador (LAO); Sistemas de adquisición de datos (SAD);Tecnologías de la Información y la Comunicación (TIC); trabajos prácticos de Física.

\section{INTRODUCCIÓN}

En los últimos tiempos, las tecnologías de la información y la comunicación (TIC) aplicadas a la educación científica han experimentado una gran evolución, tanto en el desarrollo de herramientas cada vez más potentes como en la mejora pedagógica de los contenidos del software educativo. En este área de trabajo, numerosos estudios han mostrado la utilidad didáctica de las nuevas tecnologías, como medios interactivos de comunicación que permiten el acceso a toda clase de información (textos, imágenes, tipos diferentes de datos, gráficas, etc.), como instrumentos para la resolución de ejercicios y problemas, como herramientas que efectúan simulaciones de los experimentos y de los fenómenos científicos, o para medir y controlar experimentos de laboratorio (Long, 1991, Pontes, 2005a).

De modo más específico, en el campo de la enseñanza de la ingeniería, además de las aplicaciones generales de las nuevas tecnologías que se han citado anteriormente, numerosos trabajos publicados en libros y revistas han mostrado las interesantes posibilidades educativas que ofrecen los programas de ordenador para mejorar los procesos de aprendizaje. Por ejemplo, se pueden programar tareas para explicitar los 
conocimientos previos de los alumnos, ayudándoles a sugerir sus propias hipótesis y contrastarlas con los modelos teóricos, con objeto de promover la comprensión de los conceptos científicos mediante procesos de cambio conceptual (Windschitl \& Andre, 1998). Se pueden diseñar programas-guía de actividades que ayuden a reflexionar durante la interacción del alumno con los programas de simulación y mejorar la compresión de los modelos científicos simulados. Se pueden considerar las actividades de resolución de problemas y la realización de experimentos virtuales, por parte de los alumnos, como actividades o procesos de investigación orientada (Pontes, 1999). Por último, también se pueden elaborar instrumentos de autoevaluación del conocimiento, adaptados a cada tipo de alumno, que permitan analizar el progreso seguido durante el aprendizaje de un tema y realizar diagnósticos sobre deficiencias o proponer nuevas tareas para seguir avanzando. En definitiva, creemos que las nuevas tecnologías pueden utilizarse como herramientas de reflexión donde el alumno es protagonista de la construcción del conocimiento y controlar de forma consciente su propio proceso de aprendizaje (Pontes, 2005b).

En este contexto de aplicación de las TIC a la mejora de la educación científica y tecnológica se inserta este proyecto de trabajo, que se ha centrado en el desarrollo y aplicación práctica de un sistema tutorial interactivo y un laboratorio virtual sobre el manejo de un sistema computacional de adquisición de datos experimentales conectado a un sistema de sensores físicos que actúan como instrumentos de medida en el laboratorio de Física. Este proyecto, por tanto, se puede incluir en un subdominio de la Informática Educativa, denominado Laboratorio Asistido por Ordenador (LAO) o también Experimentación Asistida por Ordenador (EXAO), donde el ordenador se considera como instrumento de laboratorio asociado a un sistema de adquisición de datos recogidos por diversos sensores, en aquellos experimentos en los que se necesitan un gran número de datos experimentales, pudiendo ser procesados además con programas del propio ordenador (Collins \& Greensalde, 1989; Cortel, 1999).

En la actualidad, los fabricantes de material de laboratorio de Física van incluyendo cada vez mayor número de equipos experimentales que llevan ordenadores acoplados, los cuales recogen y tratan los datos experimentales, a partir de los cuales se pueden realizar simulaciones, construir gráficas que muestran la relación entre variables o realizar cálculos y ajustes de diferente tipo que ayudan al estudiante en el desarrollo de la experiencia. Esta aplicación de los ordenadores puede ser muy útil en la enseñanza experimental de la Física y en la formación de ingenieros, a nivel básico y avanzado, ya que puede servir de introducción al interesante dominio de la automatización, que tiene tanta importancia en la Ingeniería moderna (Meyer, 1992; $\mathrm{Li}, 1998$ ). En los últimos años ha tenido lugar un desarrollo importante de los sistemas informatizados para la adquisición y tratamiento de datos experimentales, así como para el control de aparatos e instrumentos, porque se han desarrollado equipos potentes y asequibles que han permitido la incorporación inmediata de estos sistemas al ámbito industrial y científico. Paralelamente, también han aparecido equipos experimentales dirigidos a la enseñanza de carácter científico o técnico que han pasado a formar parte del catálogo de instrumentos de laboratorio.

Un sistema de adquisición de datos (SAD) es un dispositivo de medición que permite que los datos experimentales obtenidos con sensores de diversas magnitudes físicas 
sean leídos automáticamente, almacenados y analizados por un ordenador personal (Gil y Rodríguez, 2001). El ordenador, provisto de una interfase apropiada y de un programa de adquisición y análisis, permite a su vez procesar y monitorear en tiempo real las variables relevantes del sistema físico en estudio. En muchos laboratorios científico-técnicos, al igual que en las industrias modernas, se utilizan diversas interfases y sensores electrónicos para medir distintos parámetros de cualquier tipo. Tales sensores son los elementos que transforman la magnitud que se desea medir en una señal eléctrica. En general, cada sensor necesita un pequeño circuito auxiliar para amplificar o ajustar la señal eléctrica. Dicha señal se envía a un circuito más complejo, llamado interfaz o interfase, que la transforma en una secuencia de valores digitales de tensión que el ordenador puede leer, almacenar y procesar. El repertorio de sensores que existe en la actualidad es muy amplio en cualquier equipo experimental asociado a un SAD. De hecho, hay sensores disponibles para medir cualquier magnitud física, química, o biológica imaginable y es frecuente encontrar en los laboratorios docentes sensores de temperatura, de presión, de conductividad, de medida de $\mathrm{pH}$, de campo magnético, de intensidad luminosa, de posición, etc.

Con el fin de aprovechar algunas de las muchas posibilidades que ofrece el campo de la experimentación asistida por ordenador en la enseñanza de la física, llevamos varios años trabajando en la utilización didáctica de un sistema computacional de adquisición de datos físicos (Pontes et al., 2003). Sin embargo, hemos observado que este sistema es bastante sofisticado y no resulta fácil de manejar a nuestros alumnos, de modo que hemos considerado necesario elaborar un programa informático de simulación que ayude a los estudiantes a familiarizarse previamente con los instrumentos y técnicas que requiere la experimentación asistida por ordenador, partiendo de un prototipo inicial que hemos tratado de ampliar y mejorar. Esta actividad se ha llevado a cabo en el marco de un proyecto de innovación educativa apoyado por la Unidad de Garantía de Calidad de la Universidad de Córdoba.

En el desarrollo de este proyecto educativo convergen las aportaciones de dos líneas de trabajo diferentes: la utilización didáctica de programas de simulación para mejorar el aprendizaje de la Física (Pontes et al, 2001; Pontes, 2005b) y la utilización de programas de ordenador para controlar sistemas de adquisición de datos experimentales obtenidos mediante distintos tipos de sensores físicos (Cortel, 1999; Gil y Rodríguez, 2001). Estas líneas de trabajo, que podrían desarrollarse de forma independiente, tienen dentro de este proyecto de trabajo un hilo conductor común que es la utilización de las nuevas tecnologías informáticas en el aprendizaje de la física universitaria y en la formación científica de estudiantes de ingeniería ( $\mathrm{Li}, 1998$ ).

\section{OBJETIVOS DEL PROYECTO}

En este proyecto de innovación hemos tratado, en primer lugar, de desarrollar un programa informático de simulación que ayude a los alumnos a familiarizarse con un sistema computacional de adquisición de datos experimentales, con el fin de facilitar posteriormente el uso práctico del citado sistema en el desarrollo de experiencias de laboratorio de Física. Estos fines generales se pueden desglosar en diversos objetivos más concretos o específicos, como son los siguientes: 
- Diseñar un software interactivo destinado a integrar las nociones básicas sobre sensores y sistemas de adquisición de datos, con objeto de aplicar tales instrumentos en el desarrollo de experiencias correspondientes a los programas de física de primer curso de universidad en distintas titulaciones de los estudios de ingeniería.

- Proporcionar al programa informático un interfaz amigable, que sea de fácil uso y disponga de ayuda, que permita navegar libremente por los diferentes módulos del sistema, que sea interesante desde el punto de vista educativo y que resulte atractivo para el usuario.

- Incluir en el programa un sistema tutorial interactivo y multimedia (con hipertexto, imágenes, animaciones,...) donde se combinará el acceso a la información sobre los conceptos fundamentales sobre sensores físicos y sistemas de adquisición de datos, a nivel universitario básico, con el desarrollo de actividades orientadas a favorecer el desarrollo de destrezas experimentales y procedimientos científicos.

- Construir un laboratorio virtual en el que se llevarán a cabo los experimentos adecuados para mejorar la comprensión de los conceptos desarrollados en el sistema tutorial, junto con un editor de prácticas guiadas, donde el usuario podrá desarrollar de forma virtual una serie de montajes prácticos, con objeto de llegar a familiarizarse con el funcionamiento del sistema de adquisición de datos.

- Elaborar un conjunto de materiales didácticos que ayuden a los alumnos a realizar de forma práctica (utilizando el sistema real de adquisición de datos y los sensores correspondientes) una serie de experiencias de física, que han estudiado previamente con ayuda del programa de simulación.

- Analizar la influencia de la aplicación informática elaborada, y de los materiales didácticos complementarios, en el proceso de aprendizaje que realizan nuestros alumnos a través de las experiencias de laboratorio.

Al desarrollar estos objetivos también hemos intentado contribuir al avance y profundización de la línea de trabajo llevada a cabo por un grupo de profesores y alumnos colaboradores del Departamento de Física Aplicada de la UCO, relacionada con la elaboración de laboratorios virtuales y programas de simulación de fenómenos y experimentos físicos (Pontes et al., 2001, Pontes et al., 2003), tratando de mantener el mayor grado de coherencia con respecto a otras aplicaciones anteriores, para que los alumnos usuarios se familiaricen rápidamente con el nuevo software.

\section{FASES DE DESARROLLO DE LA EXPERIENCIA}

Hemos de indicar que al iniciar este proyecto de innovación educativa, pretendemos comenzar a desarrollar un extenso plan de trabajo que no se puede llevar a cabo en un sólo curso, ya que la problemática didáctica abordada en este proyecto es muy amplia. A medio y largo plazo se pretende renovar la metodología y los recursos didácticos que se utilizan en el desarrollo de los trabajos prácticos de física, introduciendo las nuevas tecnologías de la información (TIC) como herramientas de adiestramiento virtual $\mathrm{y}$, también, como instrumentos de recogida y análisis de datos experimentales. Por tanto, dado que la elaboración y evaluación de software educativo 
es un proceso lento y dificultoso, pretendemos realizar una serie de proyectos sucesivos, en los que vamos a ir tratando de superar diversas etapas con objetivos y tareas muy concretas, sobre la base de que los resultados de cada proyecto servirán como punto de partida para el desarrollo del siguiente. En lo que respecta a las acciones realizadas en este primer proyecto, llevado a cabo durante el curso académico 2004-05, podemos distinguir varias fases de desarrollo que se describen a continuación.

\section{Fase de preparación}

Antes de comenzar a desarrollar el proyecto se han analizado diversos programas de informática educativa, relativos a la enseñanza de la ciencia y la tecnología, con objeto de recabar información acerca de las características generales que debe poseer el software didáctico en tales dominios, mostrando especial atención por el tratamiento informático de la simulación de fenómenos y por el diseño de laboratorios virtuales. Entre tales programas se encuentran varios proyectos de fin de carrera de estudiantes de ingeniería técnica, realizados en la Escuela Politécnica Superior de la Universidad de Córdoba, que han tratado el tema de los sistemas de adquisición de datos y su utilización en el desarrollo de experiencias de Física, de Electricidad o de Electrónica. Al mismo tiempo, en esta fase preparatoria se ha realizado una revisión de trabajos sobre la problemática didáctica del aprendizaje experimental de la Física, con objeto de identificar los aspectos pedagógicos más importantes que se querían integrar en el desarrollo del sistema informático (contenidos teóricos, experiencias virtuales, tareas de desarrollo de destrezas, actividades sobre aprendizaje de procedimientos,...)

\section{Fase de elaboración del material didáctico}

Tras la fase preparatoria hemos procedido a elaborar un sistema multimedia que ayude a nuestros alumnos a comprender el funcionamiento de un sistema computacional de adquisición de datos y que, mediante el desarrollo de experiencias virtuales, les permita familiarizarse con el uso del ordenador como instrumento de laboratorio cuando se conecta a un conjunto adecuado de sensores físicos. Para desarrollar este material informático hemos partido de un prototipo inicial (Pontes et al., 2003), que ha sido ampliado y mejorado a lo largo de esta etapa. Las características del programa, junto con algunas imágenes que permitan comprender mejor la estructura del mismo, se detallarán en un apartado posterior de esta memoria.

Al mismo tiempo que se desarrollaba el programa de simulación hemos elaborado un conjunto de materiales didácticos que ayuden a los alumnos a realizar de forma práctica una serie de experiencias de Física, utilizando el sistema real de adquisición de datos y los sensores correspondientes. En el primer año de esta innovación educativa hemos elaborado un conjunto de boletines de prácticas restringido al bloque de trabajos prácticos de electricidad, una vez que los alumnos han estudiado previamente el desarrollo de las mismas con ayuda del laboratorio virtual. 


\section{Fase de aplicación}

Una vez que se ha finalizado el desarrollo del programa informático de simulación hemos tratado de utilizarlo en nuestra práctica docente, como herramienta complementaria en la programación de trabajos prácticos de física, en primer curso de ingeniería técnica de diversas especialidades de nuestro centro (en forma de experiencia piloto), siguiendo la metodología de trabajo que se describe en un apartado posterior. También hemos tratado de analizar la influencia de esta aplicación informática, y de los materiales didácticos complementarios, en el proceso de aprendizaje que realizan nuestros alumnos a través de las experiencias de laboratorio, evaluando los informes de las sesiones de trabajo realizadas con el software y el sistema de adquisición de datos experimentales.

\section{CARACTERÍSTICAS Y APLICACIONES DEL SOFTWARE}

Para desarrollar el programa informático al que nos hemos referido anteriormente (en la fase de elaboración de material didáctico) hemos partido de un prototipo inicial, elaborado previamente (Pontes et al., 2003), que en este proyecto se ha ampliado y desarrollado con mayor profundidad. El programa se ha elaborado en el lenguaje Visual Basic $6.0 \mathrm{y}$, por tanto, se trata de una aplicación informática para el entorno Windows que debe ser instalada en un ordenador personal o en una red local. Los usuarios de esta aplicación informática son nuestros propios alumnos y otros estudiantes de ciencias o ingeniería que puedan estar interesados en su utilización, siempre que dispongan en los laboratorios de sus centros de un conjunto de sensores físicos adecuados, de un sistema de adquisición de datos como el que hemos utilizado en nuestra experiencia y del software Data Studio que acompaña al citado sistema (Pasco, 2000). En el futuro tenemos previsto ir ampliando este software y realizar una versión en Visual Basic. Net que pueda ubicarse en Internet y pueda ser utilizada por un número mayor de usuarios.

\section{Estructura del programa Sensores 1.0}

La aplicación desarrollada en este proyecto, que se denomina "Sensores 1.0", está constituida por los siguientes módulos principales: Tutorial, Laboratorio Virtual, Prácticas Guiadas, Animaciones y Ayuda. Las principales características de tales módulos se describen a continuación:

\section{Tutorial}

En este módulo se tiene acceso a un documento interactivo donde el usuario puede consultar todos los temas fundamentales sobre los sistemas de adquisición de datos y los sensores de medida de un laboratorio, así como los aspectos más importantes sobre los laboratorios virtuales, las prácticas guiadas y las animaciones. Tales contenidos se exponen con un nivel de formulación conceptual que pueda ser comprendido por alumnos de los últimos cursos de bachillerato y de primer curso de la universidad. Como puede observarse en la figura 1 , en realidad se trata de un hipertexto integrado por cuatro partes diferentes que se describen a continuación. 
En primer lugar existe un Tutorial general, donde se representan, con ayuda de dibujos y esquemas, los conceptos básicos del tema y las configuraciones más conocidas sobre sistemas de adquisición de datos. A continuación se dispone de un Tutorial del laboratorio virtual, donde se presentan los objetivos de las experiencias que se implementan en el módulo de laboratorios virtuales, se exponen los fundamentos teóricos de cada experiencia simulada, se aconsejan unos pasos a seguir en la realización de cada experimento virtual $y$, por último, se exponen las conclusiones que se derivan del experimento. También existe un Tutorial de prácticas guiadas, donde se presentan los objetivos de las experiencias que se implementan en el módulo de prácticas guiadas y se explica el procedimiento que debe seguirse para realizar una práctica real con ayuda del sistema de adquisición de datos y de los sensores o elementos físicos que sean necesarios. Por último, se dispone de un Tutorial de animaciones, donde se presentan los objetivos y características de cada una de las aplicaciones prácticas de los sensores que se implementan en el módulo de animaciones, se exponen los fundamentos teóricos de cada aplicación y se explica la función de los diferentes controles relacionados con la ejecución de cada animación.

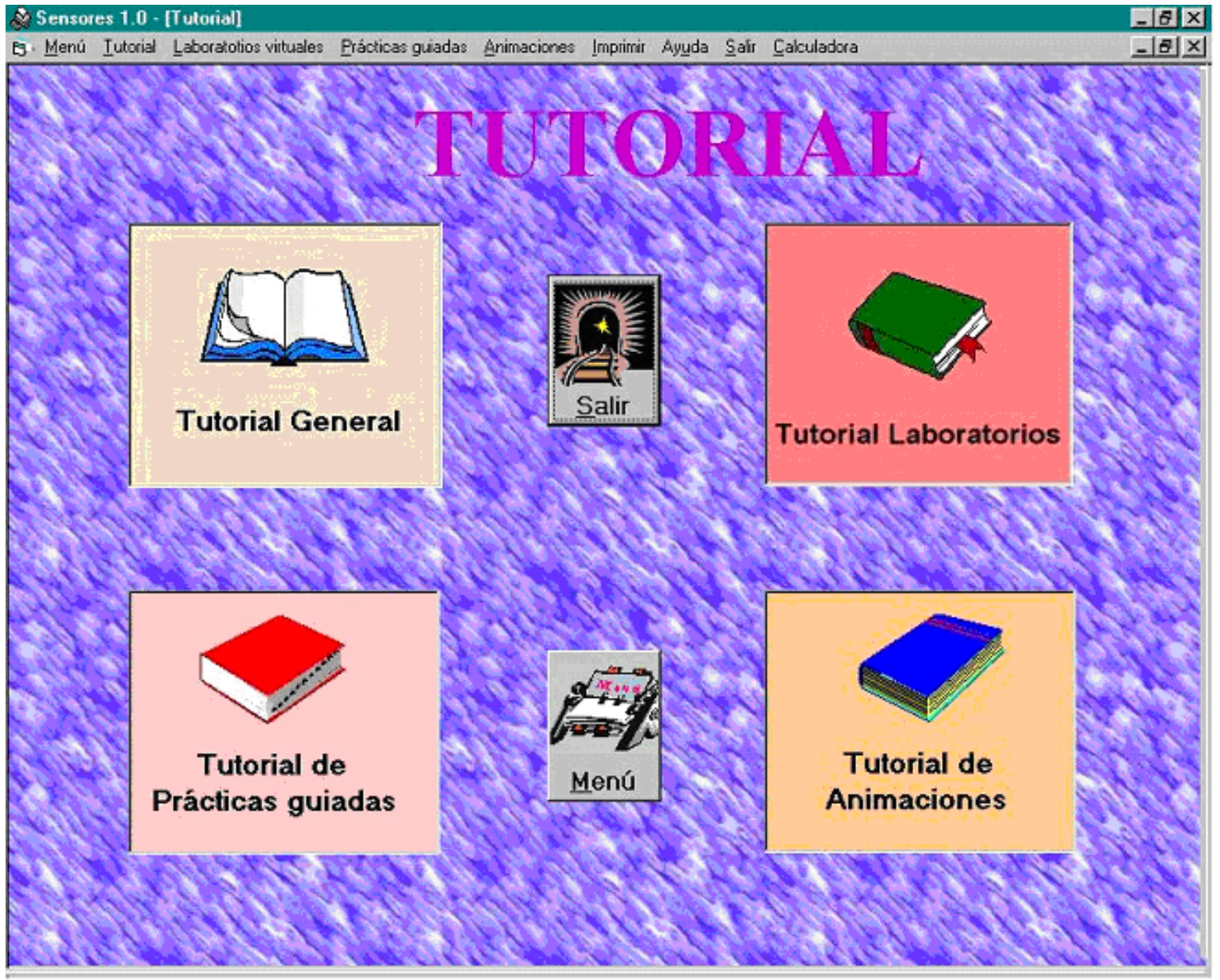

Figura 1. Ventana de acceso a las diversas partes del módulo tutorial. 


\section{Animaciones}

Para mejorar la comprensión de los conceptos desarrollados en el tutorial se hace referencia, a lo largo del módulo de Animaciones, a una serie de aspectos relacionados con el comportamiento de los sensores en la medida de diferentes magnitudes físicas. Tales fenómenos pueden simularse mediante el ordenador, como puede observarse en la figura 2, presentando animaciones que facilitarán al alumno la comprensión de los mismos, de forma visual y más atractiva que en los libros de texto. Las animaciones o simulaciones de fenómenos físicos controlados por algún tipo de sensor se incluyen en un módulo independiente al que se puede acceder desde el menú principal y desde los distintos bloques del módulo Tutoriales.

En las actividades de este módulo el programa de simulación no proporciona datos cuantitativos como ocurre en el módulo llamado laboratorio virtual, sino que se centra en proporcionar una visión animada del proceso físico y en mostrar unas relaciones de tipo gráfico que ofrecen información sobre la naturaleza del fenómeno, desde el punto de vista teórico. Finalmente, en este módulo, el desarrollo de cada animación se controla con varios botones de la parte superior de la pantalla que permiten iniciar, avanzar o detener la animación. En la figura 2 se muestra un ejemplo de animación correspondiente al fenómeno de caída y rebote en el suelo de una pelota, controlado por un sensor de movimiento situado por encima de la pelota. La gráfica adjunta representa el tipo de representación de la posición de la pelota respecto al tiempo que proporciona el sistema de adquisición de datos real disponible en nuestro laboratorio (Pasco, 2000), cuando se utiliza el software Data Studio para analizar los datos que proporciona el sensor de movimiento al citado sistema.

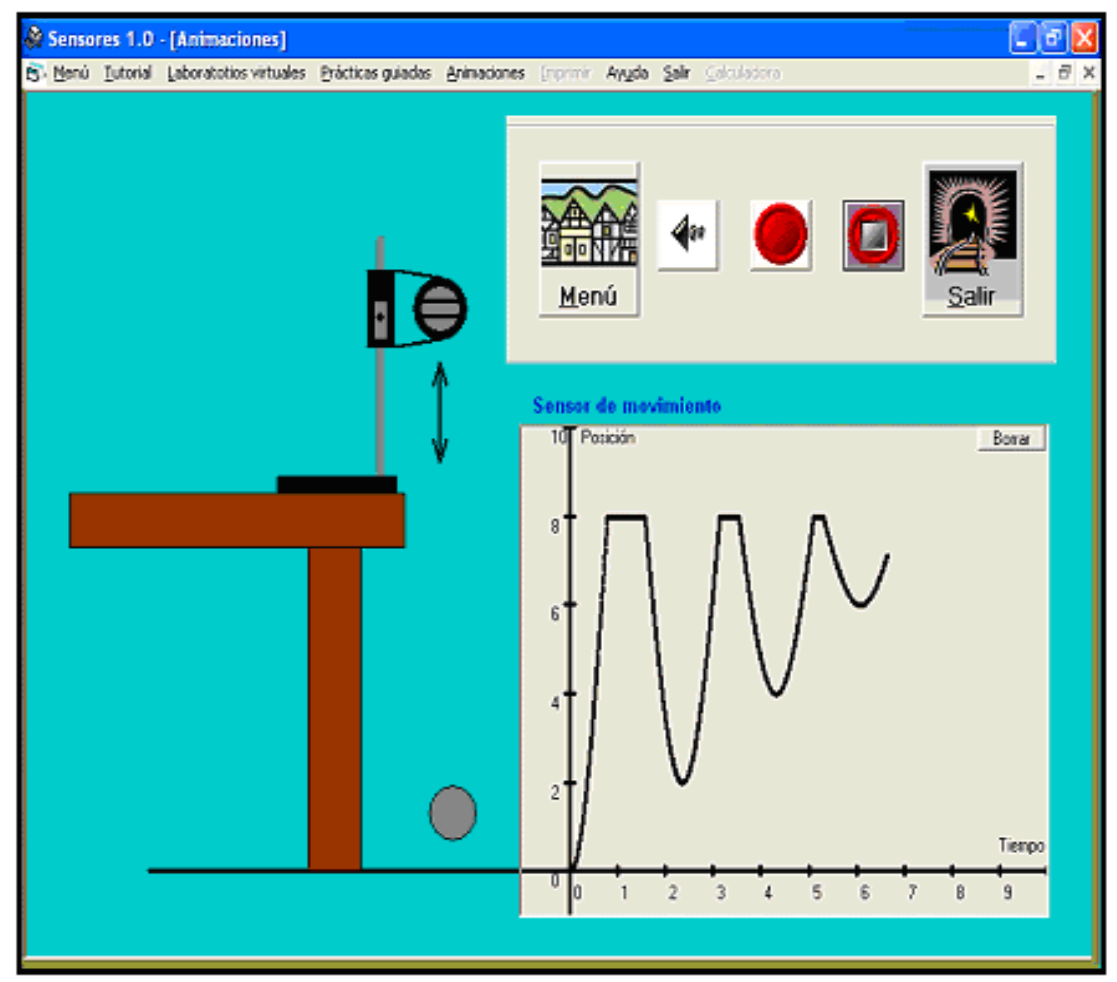

Figura 2. Ejemplo de ventana del módulo Animaciones. 


\section{Prácticas Guiadas}

En este módulo se incluyen una serie de experimentos de montaje y medida de magnitudes con sensores físicos, utilizando el sistema de adquisición de datos de la marca Pasco. En este módulo no se pretende manipular datos procedentes de prácticas simuladas, como ocurre en el módulo denominado Laboratorios Virtuales, sino que se pretende fomentar el aprendizaje de destrezas y procedimientos experimentales, tratando de familiarizar al alumno con la utilización del sistema de adquisición de datos que utilizará posteriormente en las prácticas reales. Para ello se dispone de una especie de vitrina virtual, donde existe un conjunto de materiales necesarios para realizar el montaje de una experiencia, y al lado derecho hay una ventana de trabajo que representa una mesa de laboratorio, como puede observarse en la figura 3.

El proceso a seguir en cada práctica guiada consiste en conseguir que el alumno vaya eligiendo los materiales adecuados para realizar el montaje, arrastrándolos con el ratón desde la vitrina hasta la ventana de trabajo, y tras finalizar el montaje poder realizar las mediciones necesarias con los sensores conectados al circuito diseñado. Si el alumno realiza el montaje correctamente se le permitirá realizar de forma virtual las mediciones de los parámetros del circuito que se le indiquen, mediante los sensores conectados a la interfaz del sistema de adquisición de datos. El desarrollo de la experiencia seguirá, por tanto, una secuencia similar a la que desarrollaríamos en un laboratorio real. Los iconos de la vitrina de material disponible se encuentran al principio activos (dibujados en negro), pero a medida que se van utilizando para realizar el montaje van quedando inactivos (dibujados en gris).

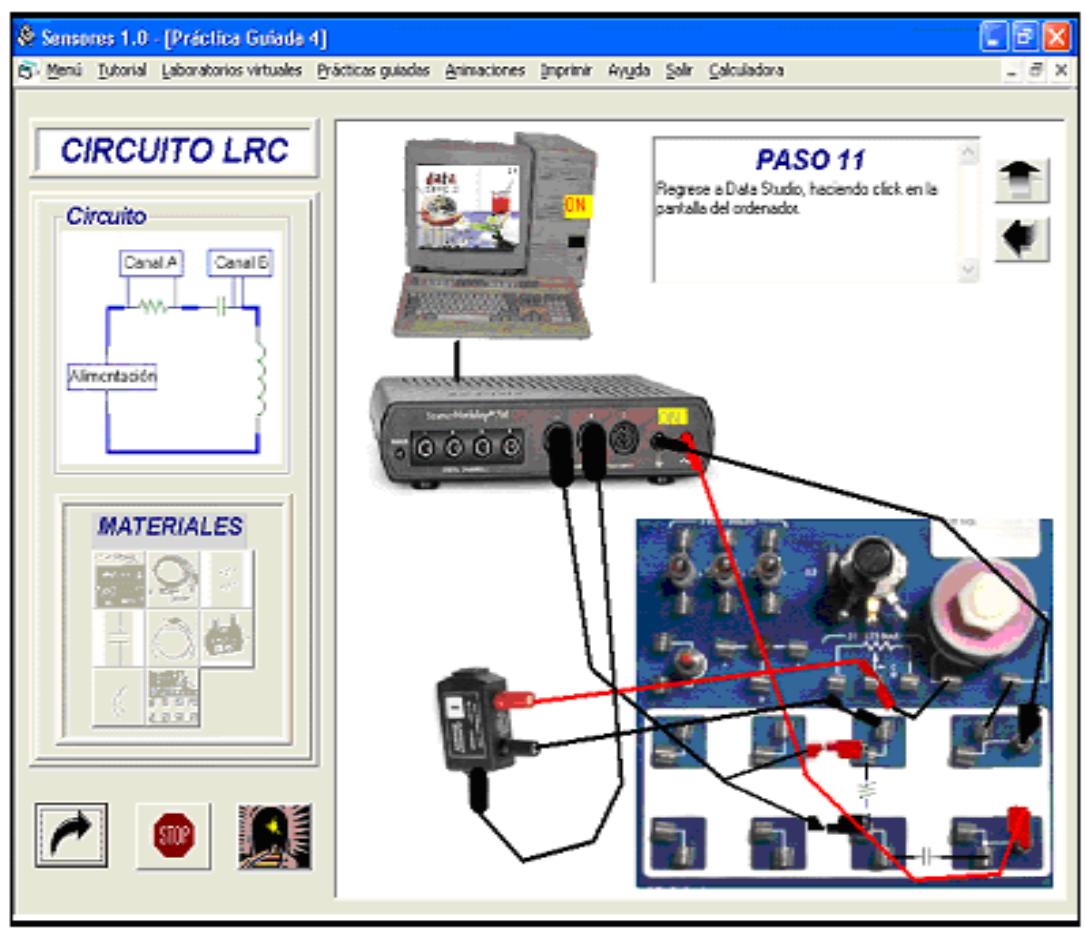

Figura 3. Ventana de trabajo del módulo Prácticas Guiadas. 
En el montaje virtual de cada experiencia el alumno puede optar por seguir las orientaciones que ofrece el programa en cada etapa hasta finalizar el montaje, o bien puede optar (sobre todo al principio) por solicitar al programa la realización del montaje de forma automática (incluyendo explicaciones sobre cada etapa). En cualquiera de tales opciones lo que se pretende es fomentar el aprendizaje de procedimientos experimentales y el adiestramiento previo en el manejo del sistema de adquisición de datos. En la figura 3 se muestra una etapa del proceso de montaje virtual de una experiencia sobre el funcionamiento del circuito RCL en corriente alterna, utilizando un sensor de voltaje (canal A) y un sensor de intensidad de corriente (canal B). En la ventana derecha de dicha figura se muestra, de forma bastante realista, la disposición final del montaje del circuito con todos los elementos que lo integran: PC con el software Data Studio, interfaz del SAD, panel de montajes (con resistencia, bobina y condensador, sensores y cables de conexión.

\section{Laboratorios Virtuales}

En este módulo se incluyen una serie de experimentos simulados, como el que se muestra en las figuras 4 y 5 , que tienen el objetivo de ayudar a los alumnos a adquirir conocimientos de carácter procedimental y a comprender mejor la naturaleza de las relaciones de tipo cuantitativo que existen entre las variables físicas, medidas con sensores y controladas por un sistema de adquisición de datos. Las experiencias simuladas se incluyen en un módulo independiente al que se puede acceder desde el menú principal o también desde el tutorial, mediante botones de enlace.

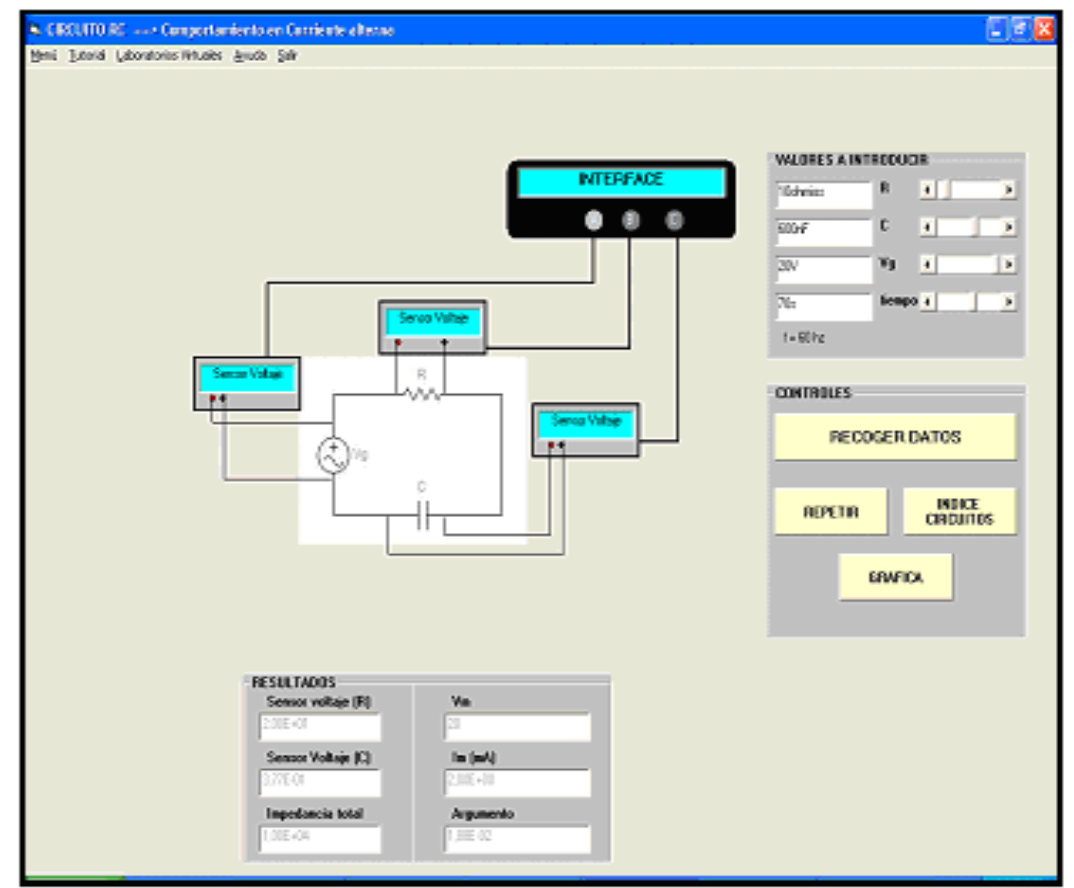

Figura 4. Simulación de un circuito $R C$ en el módulo Laboratorios Virtuales.

Dentro de este módulo se han implementado (en la primera versión del programa) una serie de experiencias virtuales relacionadas con el manejo de sensores eléctricos, utilizados en la medida de variables relacionadas con el funcionamiento de sistemas 
eléctricos y electrónicos. En estas experiencias tratamos de simular el funcionamiento de la interfaz y de los sensores de voltaje e intensidad de corriente de un equipo experimental basado en el sistema de adquisición de datos descrito anteriormente.

En cada experiencia simulada de este módulo se muestran, de forma interactiva e instantánea, los cambios que se producen en diversas magnitudes del sistema, al modificar una variable cualquiera del mismo, como puede verse en la figura 4 donde se ha simulado el comportamiento de un circuito RC en corriente alterna, incluyendo los datos de entrada (valores a introducir por el usuario) y los datos de salida (ventana de resultados) o medidas que recogen los sensores de la experiencia conectados al SAD. Desde cada experiencia simulada se pueden abrir ventanas que dan acceso a tablas de datos experimentales y a gráficos que muestran la relación funcional entre diversas variables del circuito. En la figura 5 se muestra el resultado de pulsar el botón gráficas para visualizar la relación entre voltaje e intensidad de corriente en el mismo circuito RC de corriente alterna de la figura 4.

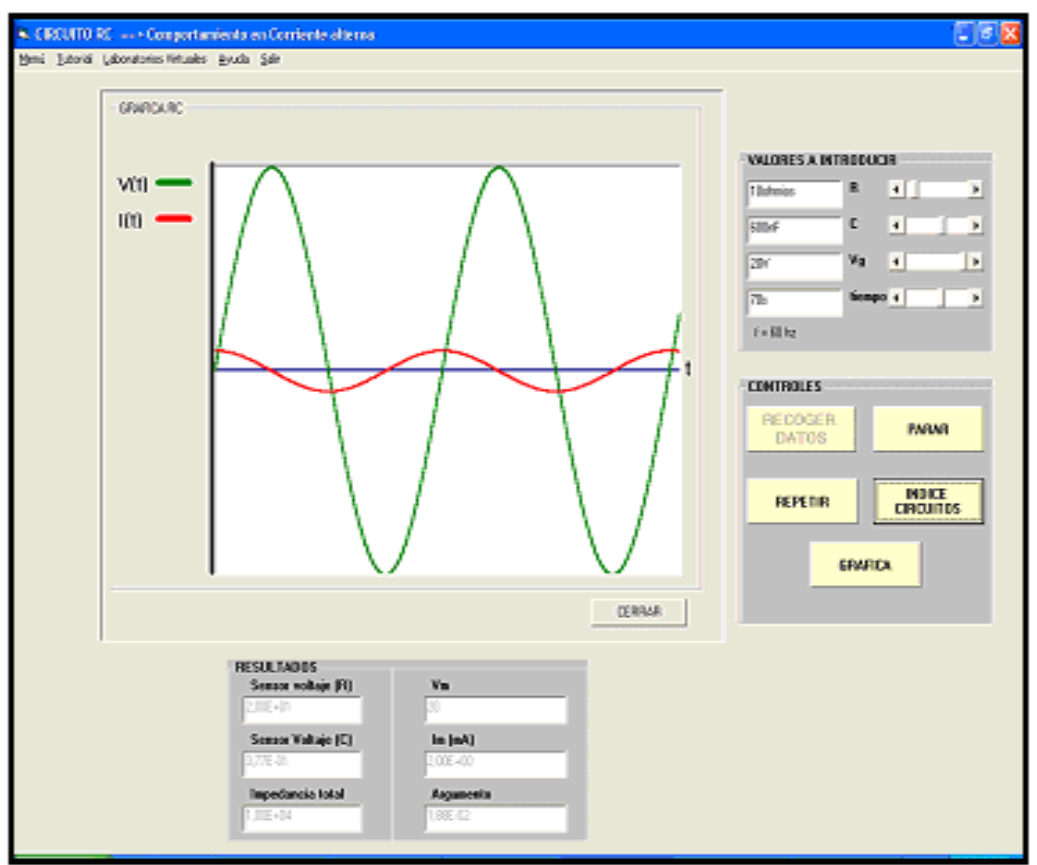

Figura 5. Gráfica $V$-I de la simulación del circuito $R C$ en corriente alterna.

En la primera versión del programa Sensores se han simulado, dentro del módulo Laboratorios virtuales, sólo experiencias relacionadas con el uso de sensores de medida de magnitudes eléctricas, con el fin de poder empezar a utilizar el programa como instrumento de adiestramiento de nuestros alumnos en el uso del sistema de adquisición de datos real. En versiones posteriores del programa se irán implementando experiencias virtuales relacionadas con el estudio de fenómenos ondulatorios, ópticos, mecánicos, térmicos o magnéticos, que requieren la utilización de una gama más amplia de sensores.

\section{Ayuda}

Además del Tutorial el usuario puede acceder desde cualquier parte del programa, a un módulo de ayuda que es también un hipertexto donde se ofrece información 
completa e interactiva sobre la estructura y uso del programa. A este módulo pueden acceder usuarios que no tengan necesariamente grandes conocimientos de informática, por lo que la estructura del mismo es bastante fácil de manejar, ya que el programa presenta el aspecto típico de un software realizado bajo entorno Windows, que está bastante estandarizado, lo cual facilita el manejo del programa por parte de todo tipo de usuarios.

\section{Metodología de utilización del software en esta experiencia}

En general el usuario de esta aplicación informática puede acceder a cada módulo del programa de forma independiente, de modo que puede utilizar el sistema de la forma que estime conveniente. Sin embargo, para obtener el mejor aprovechamiento didáctico del programa, cuando el usuario es un alumno, le aconsejamos seguir el orden que se propone a continuación:

1) Navegación inicial por el hipertexto de Ayuda para conocer la estructura del programa y la forma de utilizarlo.

2) Consulta de los contenidos en hipertexto del módulo Tutorial, tratando de familiarizarse con los conceptos básicos sobre sensores físicos y sistemas de adquisición de datos.

3) Acceso al módulo de las Animaciones que ilustran la simulación dinámica de diversos procesos en los que se utilizan sensores físicos.

4) Aprendizaje por simulación de los procesos de manejo del sistema de adquisición de datos, accediendo al módulo de Prácticas Guiadas, donde se indican todos los pasos a seguir para diseñar experimentos de carácter virtual.

5) Desarrollo de tareas de aprendizaje relacionadas con las experiencias incluidas en el módulo denominado Laboratorio virtual.

El manejo del programa de simulación del sistema de adquisición de datos se puede realizar en el propio laboratorio de Física, en las aulas de informática del centro de cálculo, o en la casa de los alumnos, ya que sólo se necesita de un ordenador personal para instalar y ejecutar el software citado. Para el análisis de los contenidos del programa y para desarrollar todas las actividades simuladas (animaciones, prácticas guiadas y experiencias incluidas en el laboratorio virtual) se pueden necesitar bastantes horas de trabajo por parte del alumnado. Pero en nuestra experiencia docente los grupos de alumnos participantes han realizado varias sesiones de trabajo de un par de horas, seleccionando para cada sesión algunas actividades concretas que ilustren previamente las experiencias reales que se van a realizar después con el sistema de adquisición de datos, los sensores y demás instrumentos requeridos en cada práctica.En cada sesión de trabajo experimental los alumnos, distribuidos en pequeños grupos, han utilizado el programa informático desarrollando las tareas recogidas en un programa-guía, que es un documento de trabajo complementario destinado a orientar la actividad que realiza el alumno con el ordenador y a favorecer la reflexión o el debate durante el proceso de aprendizaje desarrollado con ayuda del software. Por tanto, la metodología educativa que empleamos en esta experiencia es la misma que ya hemos aplicado en ocasiones anteriores utilizando otros programas 
informáticos de simulación y que se ha descrito de forma más amplia en otros trabajos anteriores (Pontes et al., 2001; Pontes, 2005b)

Tras el proceso de adiestramiento en el uso del SAD, utilizando el programa de simulación "Sensores", se forman grupos de alumnos que proceden a diseñar y realizar diversos experimentos en el laboratorio de Física, haciendo uso del sistema de adquisición de datos real y de los sensores físicos que sean necesarios para el desarrollo de cada experiencia. En tales sesiones los alumnos de cada grupo, bajo la supervisión de un profesor o de un colaborador del departamento experto en el manejo del SAD, van realizando las actividades planteadas en los boletines o programas-guía de cada experiencia (materiales complementarios), formulando hipótesis, introduciendo datos y analizando los resultados que proporciona el sistema de adquisición de datos en cada experimento.

Finalmente, los alumnos que han participado en esta primera experiencia han tenído que elaborar y presentar un informe escrito del trabajo realizado en cada sesión, donde deben sintetizar e interpretar los datos recogidos durante la experiencia y deben responder a las cuestiones planteadas en el programa-guía de actividades de cada experiencia. También han tenido que realizar una prueba escrita donde se plantean algunas preguntas que nos permiten valorar la influencia educativa de los instrumentos didácticos desarrollados en este proyecto.

\section{PRIMEROS RESULTADOS Y VALORACIÓN DE LA EXPERIENCIA}

En el desarrollo de este proyecto podemos distinguir dos tipos de resultados. Por una parte se han desarrollado diversos tipos de materiales educativos relacionados con la enseñanza experimental de la Física y el aprendizaje de destrezas científicas. Por otra parte se han aplicado tales materiales en un contexto real y hemos tratado de evaluar su influencia recogiendo unos primeros resultados sobre el proceso educativo.

Con relación a los resultados materiales o a los productos elaborados, en primer lugar se ha diseñado el programa de simulación denominado "Sensores", cuya ampliación y mejora, a partir de un prototipo inicial, constituye el fin principal del proyecto, ya que con este software se pretende mejorar el manejo de instrumentos sofisticados como los sistemas de adquisición de datos y el aprendizaje de técnicas experimentales relacionadas con tales sistemas. En segundo lugar, además del software descrito, se han elaborado diversas guías de prácticas o programas de actividades para orientar el trabajo de los alumnos durante el trabajo experimental. Tales tipos de materiales sólo tienen utilidad si se dispone del sistema de adquisición de datos experimentales y de los sensores físicos necesarios para el desarrollo de la experiencias correspondientes.

Con relación a los resultados educativos, ya hemos indicado que los alumnos tenían que presentar un informe escrito del trabajo realizado en cada sesión de laboratorio, interpretando los hechos observados y los datos recogidos en cada experiencia. El análisis de tales informes y los resultados procedentes de una prueba escrita final nos han permitido valorar la influencia educativa de los materiales didácticos que se han utilizado en este proyecto. Los datos sobre el proceso educativo recogidos con tales instrumentos no son concluyentes porque en este primer curso sólo ha participado un 
grupo reducido de alumnos de primer curso de ingeniería técnica. Sin embargo, podemos destacar algunos resultados cualitativos, de carácter provisional, que nos pueden servir para continuar avanzando con el desarrollo del proyecto en los próximos cursos.

En primer lugar hemos podido observar que el uso de este software ha contribuido a familiarizar a nuestros alumnos con el SAD y a realizar prácticas reales de física utilizando sensores, superando las dificultades de manejo del sistema que habíamos apreciado antes de desarrollar el citado software, lo cual nos ha permitido realizar una valoración cualitativa de esta innovación docente.

También hay que indicar que, en nuestra opinión, la experimentación asistida por ordenador proporciona una imagen más amplia de los hechos que se pretenden interpretar, al poder disponer en la pantalla del ordenador de gráficos que muestran la relación entre diferentes variables del fenómeno físico. Además contribuye al desarrollo de habilidades de tipo manual (montaje, medición) y capacidades de tipo intelectual o destrezas científicas (capacidad de observación y de expresión, orden, perseverancia, reconocimiento de errores, representación y análisis de datos...) que ofrecen una oportunidad de manifestarse mucho mayor que en otras actividades, como la resolución de problemas. Estos aspectos favorecen la realización de experimentos de laboratorio concebidos como pequeñas investigaciones (Sierra, 2003).

El uso del ordenador en el laboratorio también simplifica las tareas tediosas de realización de muchas mediciones, porque el sistema de adquisición de datos puede realizar las medidas de un modo extremadamente rápido, lo que facilita el acceso a experiencias inimaginables hace algunos años, o que se realizaban de un modo tan lento que resultaría insufrible para los alumnos. Realiza también la parte más pesada del trabajo experimental, que corresponde a la representación gráfica de los datos y la búsqueda de un modelo matemático (normalmente una función) que se ajuste a dichos datos. Esta característica es esencial en aquellas experiencias en las que se pretende obtener resultados de tipo cuantitativo, especialmente si se considera la breve duración disponible para las sesiones de tipo práctico.

Por último, aunque en este tipo de experiencias se emplean montajes similares a los que se utilizan en la experimentación convencional, pero sustituyendo los aparatos de medida por los sensores apropiados, sin embargo el sistema de adquisición de datos por ordenador permite medir, representar resultados y obtener modelos matemáticos a gran velocidad. Este hecho posibilita la repetición de experimentos haciendo, si es necesario, las modificaciones oportunas para ver inmediatamente sus efectos.

Por tanto, el uso del ordenador en el laboratorio permite dedicar mucho más tiempo a la discusión de los resultados, al planteamiento de hipótesis y su verificación experimental. Todas estas ventajas se incrementan cuando se utiliza un software de adiestramiento y familiarización de los alumnos con el sistema de adquisición de datos, como hemos podido observar en el desarrollo de esta experiencia educativa. 


\section{CONCLUSIONES}

En este trabajo hemos presentado una experiencia relacionada con el desarrollo de un proyecto de innovación educativa sobre el uso de las TIC en la formación científicotécnica de los estudiantes de primer curso de ingeniería técnica. Esta experiencia es la primera etapa de un proyecto más amplio, en la que se ha desarrollado una aplicación informática centrada en simular el funcionamiento de un sistema de adquisición de datos, cuando se conecta a un conjunto de sensores que sirven como instrumentos de medida de variables físicas en el laboratorio. Dicha aplicación consta de un sistema tutorial y diversos módulos de simulación que se han descrito anteriormente.

Desde el punto de vista docente esta primera aplicación se ha utilizado como instrumento de enseñanza virtual en una fase previa al uso real del citado sistema de adquisición de datos, por parte de nuestros alumnos en el desarrollo de experiencias de laboratorio, como medio para mejorar el desarrollo de destrezas científicas entre los estudiantes. La aplicación, en forma de experiencia piloto, de los materiales elaborados (software y programas de actividades) ha resultado útil para los alumnos que han participado en esta primera aplicación de la experiencia, como se deduce de la valoración que ellos mismos hacen en los informes de prácticas.

La experiencia llevada a cabo hasta ahora nos permite afirmar que la aplicación del software elaborado favorece la comprensión del funcionamiento instrumental del sistema de adquisición de datos y de los sensores que lo acompañan, de modo que los alumnos que lo han utilizado alcanzan un mayor grado de familiarización con la metodología experimental y con el instrumental, antes de pasar a utilizarlo en la realización de experiencias reales.

Creemos que esta experiencia también ha resultado útil para la formación didáctica de las personas que han participado en el proyecto, ya que la búsqueda de soluciones a las dificultades de que experimentan nuestros alumnos de ingeniería, en torno al aprendizaje de la física experimental siempre supone una perspectiva innovadora con respecto al proceso educativo. Así mismo, la elaboración de material didáctico conlleva necesariamente un proceso de estudio de trabajos previos, de reflexión y de discusión colectiva entre docentes que es poco frecuente en los departamentos universitarios. Por otra parte, dado que en este caso hemos procedido a la elaboración de un material didáctico basado en las nuevas tecnologías de la información y en el uso experimental de un sistema de adquisición de datos, ello ha requerido la colaboración de los profesores con alumnos colaboradores que son expertos en programación informática, lo cual supone la creación de un equipo de trabajo más amplio y en el que la interacción es más enriquecedora.

Estos primeros resultados nos parecen moderadamente satisfactorios y nos animan a seguir avanzando posteriormente en esta línea de trabajo, utilizando este software con muestras de alumnos más numerosas y evaluando de forma cuantitativa los resultados del proceso educativo. También trataremos de seguir aplicando esta metodología de trabajo para desarrollar nuevas experiencias que puedan integrarse en el programa de simulación y que puedan realizarse en el laboratorio con ayuda del sistema de adquisición de datos. 


\section{REFERENCIAS BIBLIOGRÁFICAS}

COLLINS, P.J. \& GREENSALDE, T.B (1989). Using the Computer as a Laboratory Instrument. The Physics Teacher, 76.

CORTEL, A. (1999). Utilización de la informática en el laboratorio. Alambique: Didáctica de las Ciencias Experimentales, 19, pp. 77-87.

GIL, S. y RODRÍGUEZ, E. (2001). Experimentos de Física usando nuevas tecnologías. Buenos Aires: Prentice Hall / Pearson.

LI, H. (1998). Information-Technology-Based Tools for Reengineering Construction Engineering Education. Computer Applications in Engineering Education, 6, pp. 15-21.

LONG, R.R. (1991). Review of Articles on Information Technology in School Science. School Science Review, 262, pp. 146-150.

MEYER, S.L. (1992). Data analysis for scientist and engineers. John Willey \& Sons, Inc., New York

PASCO SCIENTIFIC (2000). Experiments in Physics with computers and sensors. Distribuidora Prodel: Madrid.

PONTES, A. (1999). Utilización del ordenador en la enseñanza de las ciencias. Alambique: Didáctica de las Ciencias Experimentales, 19, pp. 53-64.

PONTES, A., MARTÍNEZ, M.P. y CLIMENT, M.S. (2001). Utilización didáctica de programas de simulación para el aprendizaje de técnicas de laboratorio en ciencias experimentales. Anales de Química. 97(3), pp.44-54.

PONTES, A., LEÓN, J., MARTÍNEZ, M.P. y CLIMENT, M.S. (2003). El uso del ordenador como instrumento para enseñar a manejar sistemas de adquisición de datos experimentales. XI Congreso Universitario sobre Innovación Educativa en las Enseñanzas Técnicas. UPC: Vilanova i la Geltru.

PONTES, A. (2005a). Aplicaciones de las nuevas tecnologías de la información en la educación científica. $1^{a}$ parte: funciones y recursos. Revista Eureka sobre Enseñanza y Divulgación de las Ciencias, 2(1), pp. 2-18.

PONTES, A. (2005b): Aplicaciones de las nuevas tecnologías de la información en la educación científica. 2a Parte: Aspectos metodológicos. Revista Eureka sobre Enseñanza y Divulgación de las Ciencias, 2(3), pp. 302-315.

SIERRA, J.L. (2003). Estudio de la influencia de un entorno de simulación por ordenadores el aprendizaje por investigación de la Física en Bachillerato. Tesis Doctoral. Universidad de Granada.

WINDSCHITL, M. y ANDRE, T. (1998). Using computer simulations to enhance conceptual change: the roles of constructivist instruction and student epistemological beliefs. Journal of Research in Science Teaching. 35(2), pp.145160. 


\section{SUMMARY}

In this article we describe an educative experience on the use of information and communication technologies in the learning of experimental techniques. It is an important topic for the development of practical physics works, in the university studies of science and engineering. The experience has been based on the design and application of a simulation program which tries to improve the learning of scientific procedures, related with the collection and later analysis of experimental data, using a computer system of data acquisition which is connected, in each experiment, to several physical sensors.

Key words: information and communication technologies; learning of experimental techniques; practical physics works simulation programs; system of data acquisition. 